\title{
A New Approach on Helices in Pseudo-Riemannian Manifolds
}

\author{
Evren Zıplar, ${ }^{1}$ Yusuf Yaylı, ${ }^{2}$ and İsmail Gök ${ }^{2}$ \\ ${ }^{1}$ Department of Mathematics, Faculty of Science, ÇankırıKaratekin University, 18000 Çankırı, Turkey \\ ${ }^{2}$ Department of Mathematics, Faculty of Science, Ankara University, 06100 Ankara, Turkey
}

Correspondence should be addressed to Evren Ziplar; evrenziplar@karatekin.edu.tr

Received 7 October 2013; Accepted 16 February 2014; Published 19 March 2014

Academic Editor: Sining Zheng

Copyright (C) 2014 Evren Ziplar et al. This is an open access article distributed under the Creative Commons Attribution License, which permits unrestricted use, distribution, and reproduction in any medium, provided the original work is properly cited.

\begin{abstract}
A proper curve $\alpha$ in the $n$-dimensional pseudo-Riemannian manifold $(M, g)$ is called a $V_{n}$-slant helix if the function $g\left(V_{n}, X\right)$ is a nonzero constant along $\alpha$, where $X$ is a parallel vector field along $\alpha$ and $V_{n}$ is $n$th Frenet frame. In this work, we study such curves and give important characterizations about them.
\end{abstract}

\section{Introduction}

Curves theory is an essential structure in the differential geometry works. Helix is one of the most spectacular curves because of its helical structure in nature and science. Helices are used in the field of imitation of kinematic motion or the shape of DNA and carbon nanotubes. Moreover, the helical structure can be seen in fractal geometry, especially in hyperhelices [1,2].

Furthermore, "helices share common origins in the geometries of the platonic solids, with inherent hierarchical potential that is typical of biological structures. The helices provide an energy-efficient solution to close-packing in molecular biology, a common motif in protein construction, and a readily observable pattern at many size levels throughout the body. The helices are described in a variety of anatomical structures, suggesting their importance to structural biology and manual therapy" [3].

A curve of constant slope or general helix in Euclidean 3 -space $E^{3}$ is defined by the property that its tangent vector field makes a constant angle with a fixed straight line (the axis of general helix). A classical result stated by Lancret in 1802 and first proved by de Saint Venant in $1845[4,5]$ as follows: A necessary and sufficient condition for a curve to be a general helix is that the ratio of the first curvature to the second curvature should be constant ; that is, $\kappa / \tau$ is constant along the curve, where $\kappa$ and $\tau$ denote the first and second curvatures of the curve, respectively. In [6], Özdamar and Hacisalihoğlu defined harmonic curvature functions $H_{i}(1 \leq i \leq n-2)$ of a curve $\alpha$ and generalized helices in $E^{3}$ to those in $n$-dimensional Euclidean space $E^{n}$. Moreover, they gave a characterization for the inclined curves in $E^{n}$ :

"A curve is an inclined curve

$$
\text { if and only if } \sum_{i=1}^{n} H_{i}^{2}=\text { constant." }
$$

Harmonic curvature functions have important role in characterizations of general helices in higher dimensions, because the notion of a general helix can be generalized to higher dimension in different ways. However, these ways are not easy to show which curves are general helices and finding the axis of a general helix is complicated in higher dimension. Thanks to harmonic curvature functions, we can easily obtain the axis of such curves. Moreover, this way is confirmed in 3dimensional spaces.

Izumiya and Takeuchi defined a new kind of helix (slant helix) and they gave a characterization of slant helices in Euclidean 3-space $E^{3}$ [7]. In 2008, Önder et al. defined a new kind of slant helix in Euclidean 4 -space $E^{4}$ which is called $B_{2}$-slant helix and they gave some characterizations of these slant helices in Euclidean 4-space $E^{4}$ [8]. And then in 2009 Gök et al. generalized $B_{2}$-slant helix in $E^{4}$ to $E^{n}, n>3$, called $V_{n}$-slant helix in Euclidean and Minkowski $n$-space $[9,10]$. Lots of authors in their papers have investigated inclined curves and slant helices using the harmonic curvature functions in Euclidean and Minkowski $n$-space [11-15]. But, 
Şenol et al. [16] see for the first time that the characterization of inclined curves and slant helices in (1) is true only for the case necessity but not true for the case sufficiency in Euclidean $n$-space. Then, they consider the precharacterizations about inclined curves and slant helices and restructure them with the necessary and sufficient condition [16].

Similar to the work in [16], in this work, we define $V_{n}{ }^{-}$ slant helix and give characterizations about the helix with necessary and sufficient condition in $n$-dimensional pseudoRiemannian manifolds for the first time.

\section{Preliminaries}

In this section, we give some basic definitions from differential geometry.

Definition 1. A metric tensor $g$ on a smooth manifold $M$ is a symmetric nondegenerate $(0,2)$ tensor field on $M$.

In other words, $g(X, Y)=g(Y, X)$ for all $X, Y \in T M$ (tangent bundle) and at each point $p$ of $M$ if $g\left(X_{p}, Y_{p}\right)=0$ for all $Y_{p} \in T_{p}(M)$; then $X_{p}=0$ (nondegenerate), where $T_{p}(M)$ is the tangent space of $M$ at the point $p$ and $g$ :

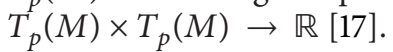

Definition 2. A pseudo-Riemannian manifold (or semiRiemannian manifold) is a smooth manifold $M$ furnished with a metric tensor $g$. That is, a pseudo-Riemannian manifold is an ordered pair $(M, g)[17]$.

Definition 3. One will recall the notion of a proper curve of order $n$ in $n$-dimensional pseudo-Riemannian manifold $M$ with the metric tensor $g$. Let $\alpha: I \rightarrow M$ be a nonnull curve in $M$ parametrized by the arc length $s$, where $I$ is an open interval of the real line $\mathbb{R}$. One denotes the tangent vector field of $\alpha$ by $V_{1}$. One assumes that $\alpha$ satisfies the following Frenet formula:

$$
\begin{aligned}
& \nabla_{V_{1}} V_{1}=k_{1} V_{2}, \\
& \nabla_{V_{1}} V_{i}=-\varepsilon_{i-2} \varepsilon_{i-1} k_{i-1} V_{i-1}+k_{i} V_{i+1}, \quad 1<i<n, \\
& \nabla_{V_{1}} V_{n}=-\varepsilon_{n-2} \varepsilon_{n-1} k_{n-1} V_{n-1},
\end{aligned}
$$

where

$$
\begin{aligned}
k_{1} & =\left\|\nabla_{V_{1}} V_{1}\right\|>0, \\
k_{i} & =\left\|\nabla_{V_{1}} V_{i}+\varepsilon_{i-2} \varepsilon_{i-1} k_{i-1} V_{i-1}\right\|>0, \quad 2 \leq i \leq n-1, \\
\varepsilon_{j-1} & =g\left(V_{j}, V_{j}\right)(= \pm 1), \quad 1 \leq j \leq n, \text { on } I,
\end{aligned}
$$

and $\nabla$ is Levi-Civita connection of $M$.

One calls such a curve a proper curve of order $n, k_{i}(1 \leq$ $i \leq n-1)$ its $i$ th curvature, and $V_{1}, \ldots, V_{n}$ its Frenet Frame field.

Moreover, let one recall that $\|X\|=\sqrt{|g(X, X)|}$ for $X \in$ $T M$, where $T M$ is the tangent bundle of $M$ [18].

\section{3. $V_{n}$-Slant Helices and Their Harmonic Curvature Functions}

In this section, we give definition of a $V_{n}$-slant helix curve in a $n$-dimensional pseudo-Riemannian manifold. Furthermore, we give characterizations by using harmonic curvatures for $V_{n}$-slant helices.

Definition 4. Let $M$ be a $n$-dimensional pseudo-Riemannian manifold and let $\alpha(s)$ be a proper curve of order $n$ (nonnull) with the curvatures $k_{i}(i=1, \ldots, n-1)$ in $M$. Then, harmonic curvature functions of $\alpha$ are defined by

$$
H_{i}^{*}: I \subset \mathbb{R} \longrightarrow \mathbb{R}
$$

along $\alpha$ in $M$, where

$$
\begin{aligned}
& H_{0}^{*}=0, \\
& H_{1}^{*}=\varepsilon_{n-3} \varepsilon_{n-2} \frac{k_{n-1}}{k_{n-2}}, \\
& H_{i}^{*}=\left(k_{n-i} H_{i-2}^{*}-\nabla_{V_{1}} H_{i-1}^{*}\right) \frac{\varepsilon_{n-(i+2)} \varepsilon_{n-(i+1)}}{k_{n-(i+1)}}, \quad 2 \leq i \leq n-2 .
\end{aligned}
$$

Note that $\nabla_{V_{1}} H_{i-1}^{*}=V_{1}\left(H_{i-1}^{*}\right)=H_{i-1}^{* \prime}$.

Definition 5. Let $(M, g)$ be a $n$-dimensional pseudoRiemannian manifold and let $\alpha(s)$ be a proper curve of order $n$ (nonnull). One calls $\alpha$ as a $V_{n}$-slant helix in $M$ if the function

$$
g\left(V_{n}, X\right)
$$

is a nonzero constant along $\alpha$ and $X$ is a parallel vector field along $\alpha$ in $M$; that is, $\nabla_{V_{1}} X=0$. Here, $V_{n}$ is $n$th Frenet frame field and $X \in T M$. Also, $X$ is called the axis of $\alpha$.

Lemma 6. Let $(M, g)$ be an-dimensional pseudo-Riemannian manifold and let $\alpha(s)$ be a proper curve of order $n$ (nonnull). Let one assume that $H_{n-2}^{*} \neq 0$ for $i=n-2$. Then, $\varepsilon_{n-3} H_{1}^{* 2}+$ $\varepsilon_{n-4} H_{2}^{* 2}+\cdots+\varepsilon_{0} H_{n-2}^{* 2}$ is nonzero constant if and only if $V_{1}\left(H_{n-2}^{*}\right)=H_{n-2}^{* \prime}=k_{1} H_{n-3}^{*}$, where $V_{1}$ and $\left\{H_{1}^{*}, \ldots, H_{n-2}^{*}\right\}$ are the unit tangent vector field and the harmonic curvatures of $\alpha$, respectively.

Proof. First, we assume that $\varepsilon_{n-3} H_{1}^{* 2}+\varepsilon_{n-4} H_{2}^{* 2}+\cdots+\varepsilon_{0} H_{n-2}^{* 2}$ is nonzero constant. Consider the following functions given in Definition 4:

$$
H_{i}^{*}=\left(k_{n-i} H_{i-2}^{*}-H_{i-1}^{* \prime}\right) \frac{\varepsilon_{n-(i+2)} \varepsilon_{n-(i+1)}}{k_{n-(i+1)}}
$$

for $3 \leq i \leq n-2$. So, from the equality, we can write

$$
k_{n-(i+1)} H_{i}^{*}=\varepsilon_{n-(i+2)} \varepsilon_{n-(i+1)}\left(k_{n-i} H_{i-2}^{*}-H_{i-1}^{* \prime}\right) \text {. }
$$

Hence, in (8), if we take $i+1$ instead of $i$, we get

$$
\begin{aligned}
\varepsilon_{n-(i+3)} \varepsilon_{n-(i+2)} H_{i}^{* \prime}= & \varepsilon_{n-(i+3)} \varepsilon_{n-(i+2)} k_{n-(i+1)} H_{i-1}^{*} \\
& -k_{n-(i+2)} H_{i+1}^{*}, \quad 2 \leq i \leq n-3
\end{aligned}
$$


together with

$$
H_{1}^{* \prime}=-\frac{1}{\varepsilon_{n-4} \varepsilon_{n-3}} k_{n-3} H_{2}^{*}
$$

or

$$
H_{1}^{* \prime}=-\varepsilon_{n-4} \varepsilon_{n-3} k_{n-3} H_{2}^{*} .
$$

On the other hand, since $\varepsilon_{n-3} H_{1}^{* 2}+\varepsilon_{n-4} H_{2}^{* 2}+\cdots+\varepsilon_{0} H_{n-2}^{* 2}$ is constant, we have

$$
\varepsilon_{n-3} H_{1}^{*} H_{1}^{* \prime}+\varepsilon_{n-4} H_{2}^{*} H_{2}^{* \prime}+\cdots+\varepsilon_{0} H_{n-2}^{*} H_{n-2}^{* \prime}=0
$$

and so

$$
\begin{aligned}
\varepsilon_{0} H_{n-2}^{*} H_{n-2}^{* \prime}= & -\varepsilon_{n-3} H_{1}^{*} H_{1}^{* \prime} \\
& -\varepsilon_{n-4} H_{2}^{*} H_{2}^{* \prime}-\cdots-\varepsilon_{1} H_{n-3}^{*} H_{n-3}^{* \prime} .
\end{aligned}
$$

By using (9) and (11), we obtain

$$
\begin{aligned}
H_{1}^{*} H_{1}^{* \prime}= & -\varepsilon_{n-4} \varepsilon_{n-3} k_{n-3} H_{1}^{*} H_{2}^{*} \\
\varepsilon_{n-(i+3)} \varepsilon_{n-(i+2)} H_{i}^{*} H_{i}^{* \prime}= & \varepsilon_{n-(i+3)} \varepsilon_{n-(i+2)} k_{n-(i+1)} H_{i-1}^{*} H_{i}^{*} \\
& -k_{n-(i+2)} H_{i}^{*} H_{i+1}^{*}, \quad 2 \leq i \leq n-3 .
\end{aligned}
$$

Therefore, by using (13), (14), and (15), an algebraic calculus shows that

$$
\varepsilon_{0} H_{n-2}^{*} H_{n-2}^{* \prime}=\varepsilon_{0} k_{1} H_{n-3}^{*} H_{n-2}^{*}
$$

or

$$
H_{n-2}^{*} H_{n-2}^{* \prime}=k_{1} H_{n-3}^{*} H_{n-2}^{*} .
$$

Since $H_{n-2}^{*} \neq 0$, we get the relation

$$
H_{n-2}^{* \prime}=k_{1} H_{n-3}^{*} .
$$

Conversely, we assume that

$$
H_{n-2}^{* \prime}=k_{1} H_{n-3}^{*} .
$$

By using (19) and $H_{n-2}^{*} \neq 0$, we can write

$$
H_{n-2}^{*} H_{n-2}^{* \prime}=k_{1} H_{n-2}^{*} H_{n-3}^{*} \text {. }
$$

From (15), we have the following equation system:

$$
\text { for } i=n-3, \quad \begin{aligned}
\varepsilon_{1} H_{n-3}^{*} H_{n-3}^{* \prime}= & \varepsilon_{1} k_{2} H_{n-4}^{*} H_{n-3}^{*} \\
& -\varepsilon_{0} k_{1} H_{n-3}^{*} H_{n-2}^{*},
\end{aligned}
$$

for $i=n-4, \quad \varepsilon_{2} H_{n-4}^{*} H_{n-4}^{* \prime}=\varepsilon_{2} k_{3} H_{n-5}^{*} H_{n-4}^{*}$

$$
-\varepsilon_{1} k_{2} H_{n-4}^{*} H_{n-3}^{*} \text {, }
$$

for $i=n-5, \quad \varepsilon_{3} H_{n-5}^{*} H_{n-5}^{* \prime}=\varepsilon_{3} k_{4} H_{n-6}^{*} H_{n-5}^{*}$

$$
-\varepsilon_{2} k_{3} H_{n-5}^{*} H_{n-4}^{*} \text {, }
$$

for $i=2, \quad \varepsilon_{n-4} H_{2}^{*} H_{2}^{* \prime}=\varepsilon_{n-4} k_{n-3} H_{1}^{*} H_{2}^{*}-\varepsilon_{n-5} k_{n-4} H_{2}^{*} H_{3}^{*}$.
Moreover, from (14) and (20), we obtain

$$
\begin{gathered}
\varepsilon_{n-3} H_{1}^{*} H_{1}^{* \prime}=-\varepsilon_{n-4} k_{n-3} H_{1}^{*} H_{2}^{*}, \\
\varepsilon_{0} H_{n-2}^{*} H_{n-2}^{* \prime}=\varepsilon_{0} k_{1} H_{n-2}^{*} H_{n-3}^{*} .
\end{gathered}
$$

So, by using the above equation system and considering (22), an algebraic calculus shows that

$$
\varepsilon_{n-3} H_{1}^{*} H_{1}^{* \prime}+\varepsilon_{n-4} H_{2}^{*} H_{2}^{* \prime}+\cdots+\varepsilon_{0} H_{n-2}^{*} H_{n-2}^{* \prime}=0 .
$$

And, by integrating (23), we can easily say that

$$
\varepsilon_{n-3} H_{1}^{* 2}+\varepsilon_{n-4} H_{2}^{* 2}+\cdots+\varepsilon_{0} H_{n-2}^{* 2}
$$

is a nonzero constant. This completes the proof.

Proposition 7. Let $(M, g)$ be a n-dimensional pseudoRiemannian manifold and let $\alpha(s)$ be a proper curve of order $n$ (nonnull). If $\alpha$ is $V_{n}$-slant helix in $M$, then we have

$$
g\left(V_{n-(i+1)}, X\right)=H_{i}^{*} g\left(V_{n}, X\right), \quad i=0,1, \ldots, n-2,
$$

where $X$ is the axis of $\alpha$. Here, $\left\{V_{1}, V_{2}, \ldots, V_{n}\right\}$ denote the Frenet frame of $\alpha$ and $\left\{H_{1}^{*}, H_{2}^{*}, \ldots, H_{n-2}^{*}\right\}$ denote the harmonic curvature functions of the curve $\alpha$.

Proof. We will use the induction method. Let $i=1$. Since $X$ is the axis of the $V_{n}$-slant helix $\alpha$, we get

$$
X=\lambda_{1} V_{1}+\cdots+\lambda_{n} V_{n}
$$

From the definition of $V_{n}$-slant helix, we have

$$
g\left(V_{n}, X\right)=\lambda_{n} \varepsilon_{n-1}=\text { constant. }
$$

A differentiation in (27) and the Frenet formulas gives us

$$
g\left(V_{n-1}, X\right)=0
$$

Again, differentiation in (28) and the Frenet formulas give

$$
\begin{gathered}
g\left(\nabla_{V_{1}} V_{n-1}, X\right)=0, \\
-\varepsilon_{n-3} \varepsilon_{n-2} k_{n-2} g\left(V_{n-2}, X\right)+k_{n-1} g\left(V_{n}, X\right)=0, \\
g\left(V_{n-2}, X\right)=\varepsilon_{n-3} \varepsilon_{n-2} \frac{k_{n-1}}{k_{n-2}} g\left(V_{n}, X\right), \\
g\left(V_{n-2}, X\right)=H_{1}^{*} g\left(V_{n}, X\right),
\end{gathered}
$$

respectively. Hence, it is shown that (25) is true for $i=1$.

We now assume that (25) is true for the first $i-1$. Then, we have

$$
g\left(V_{n-i}, X\right)=H_{i-1}^{*} g\left(V_{n}, X\right)
$$

A differentiation in (30) and the Frenet formulas give us that

$$
-\varepsilon_{n-i-2} \varepsilon_{n-i-1} k_{n-i-1} g\left(V_{n-i-1}, X\right)+k_{n-i} g\left(V_{n-i+1}, X\right)
$$$$
=\nabla_{V_{1}} H_{i-1}^{*} g\left(V_{n}, X\right) \text {. }
$$ 
Since we have the induction hypothesis, $g\left(V_{n-i+1}, X\right)=$ $H_{i-2}^{*} g\left(V_{n}, X\right)$, we get

$$
\begin{aligned}
& \left(k_{n-i} H_{i-2}^{*}-\nabla_{V_{1}} H_{i-1}^{*}\right) \frac{\varepsilon_{n-(i+2)} \varepsilon_{n-(i+1)}}{k_{n-(i+1)}} g\left(V_{n}, X\right) \\
& \quad=g\left(V_{n-(i+1)}, X\right),
\end{aligned}
$$

which gives

$$
g\left(V_{n-(i+1)}, X\right)=H_{i}^{*} g\left(V_{n}, X\right) .
$$

Theorem 8. Let $(M, g)$ be a n-dimensional pseudoRiemannian manifold and let $\alpha(s)$ be a proper curve of order $n$ (nonnull). Then, $\alpha$ is a $V_{n}$-slant helix in $M$ if and only if it satisfies that

$$
\sum_{i=1}^{n-2} \varepsilon_{n-(i+2)} H_{i}^{* 2}
$$

is equal to nonzero constant and $H_{n-2}^{*} \neq 0$.

Proof. Suppose $\alpha$ to be a $V_{n}$-slant helix. According to Definition 5 and the proof of Proposition 7,

$$
g\left(V_{n}, X\right)=\lambda_{n} \varepsilon_{n-1}=\text { constant, }
$$

where $X$ is the axis of $\alpha$. From Proposition 7, we have

$$
g\left(V_{n-(i+1)}, X\right)=H_{i}^{*} g\left(V_{n}, X\right)
$$

for $1 \leq i \leq n-2$. Moreover, from (35) and Frenet formulas, we can write

$$
-\varepsilon_{n-2} \varepsilon_{n-1} k_{n-1} g\left(V_{n-1}, X\right)=0 .
$$

Since $-\varepsilon_{n-2} \varepsilon_{n-1} k_{n-1}$ is different from zero, $g\left(V_{n-1}, X\right)=0$. It is known that the system $\left\{V_{1}, \ldots, V_{n}\right\}$ is a basis of $\varkappa(M)$ (tangent bundle) along $\alpha$. Hence, $X$ can be expressed in the form

$$
X=\sum_{i=1}^{n} \lambda_{i} V_{i}
$$

Moreover, from (38), we get the system

$$
\begin{gathered}
\varepsilon_{0} \lambda_{1}=g\left(X, V_{1}\right), \\
\varepsilon_{1} \lambda_{2}=g\left(X, V_{2}\right), \\
\vdots \\
\varepsilon_{n-3} \lambda_{n-2}=g\left(X, V_{n-2}\right), \\
\varepsilon_{n-2} \lambda_{n-1}=g\left(X, V_{n-1}\right)=0, \\
\varepsilon_{n-1} \lambda_{n}=g\left(X, V_{n}\right)
\end{gathered}
$$

by using the metric $g$. Therefore, from Proposition 7 and the above system, we can see that the following system is true:

$$
\begin{aligned}
& \lambda_{1}=g\left(X, V_{1}\right)=\varepsilon_{0} H_{n-2}^{*} g\left(X, V_{n}\right), \\
& \lambda_{2}=g\left(X, V_{2}\right)=\varepsilon_{1} H_{n-3}^{*} g\left(X, V_{n}\right),
\end{aligned}
$$

$$
\begin{aligned}
\lambda_{n-2} & =g\left(X, V_{n-2}\right)=\varepsilon_{n-3} H_{1}^{*} g\left(X, V_{n}\right), \\
\lambda_{n-1} & =g\left(X, V_{n-1}\right)=0, \\
\lambda_{n} & =\varepsilon_{n-1} g\left(X, V_{n}\right) .
\end{aligned}
$$

Thus, the axis of the curve $\alpha$ can be easily obtained as

$$
X=g\left(X, V_{n}\right)\left\{\sum_{i=1}^{n-2} H_{i}^{*} V_{n-(i+1)} \varepsilon_{n-(i+2)}+\left(\varepsilon_{n-1} V_{n}\right)\right\}
$$

by making use of equality (38) and the last system.

Therefore, from (41), we can write

$$
g(X, X)=\left[g\left(X, V_{n}\right)\right]^{2}\left(\varepsilon_{0}^{3} H_{n-2}^{* 2}+\cdots+\varepsilon_{n-3}^{3} H_{1}^{* 2}+\varepsilon_{n-1}^{3}\right) .
$$

Moreover, by the definition of metric tensor, we have

$$
|g(X, X)|=\|X\|^{2}
$$

Since $\alpha$ is a $V_{n}$-slant helix, $\|X\|=$ constant and $g\left(X, V_{n}\right)$ is nonzero constant along $\alpha$. Hence, from (42), we obtain that

$$
\varepsilon_{0}^{3} H_{n-2}^{* 2}+\cdots+\varepsilon_{n-3}^{3} H_{1}^{* 2}+\varepsilon_{n-1}^{3}
$$

is constant. In other words,

$$
\varepsilon_{0} H_{n-2}^{* 2}+\cdots+\varepsilon_{n-3} H_{1}^{* 2}=\sum_{i=1}^{n-2} \varepsilon_{n-(i+2)} H_{i}^{* 2}
$$

is constant.

Now, we will show that $H_{n-2}^{*} \neq 0$. We assume that $H_{n-2}^{*}=$ 0 . Then, for $i=n-2$ in (36), we have

$$
g\left(V_{1}, X\right)=H_{n-2}^{*} g\left(X, V_{n}\right)=0
$$

If we take derivative in each part of (46) in the direction $V_{1}$ on $M$, then we have

$$
g\left(\nabla_{V_{1}} V_{1}, X\right)+g\left(V_{1}, \nabla_{V_{1}} X\right)=0 .
$$

On the other hand, $\nabla_{V_{1}} X=0$ since $\alpha$ is a $V_{n}$-slant helix. Then, from (47), we have

$$
g\left(\nabla_{V_{1}} V_{1}, X\right)=k_{1} g\left(V_{2}, X\right)=0
$$

by using the Frenet formulas. Since $k_{1}$ is positive, it must be $g\left(V_{2}, X\right)=0$. Now, for $i=n-3$ in (36),

$$
g\left(V_{2}, X\right)=H_{n-3}^{*} g\left(V_{n}, X\right) .
$$


Since $g\left(V_{2}, X\right)=0$ and $g\left(V_{n}, X\right) \neq 0$, it must be $H_{n-3}^{*}=0$. Continuing this process, we get $H_{1}^{*}=0$. Let us recall that $H_{1}^{*}=\varepsilon_{n-3} \varepsilon_{n-2}\left(k_{n-1} / k_{n-2}\right)$; thus we have a contradiction because all the curvatures are nowhere zero. Consequently, $H_{n-2}^{*} \neq 0$.

Conversely, we assume that $\sum_{i=1}^{n-2} \varepsilon_{n-(i+2)} H_{i}^{* 2}=$ constant and $H_{n-2}^{*} \neq 0$. We take the vector field

$$
X=\lambda_{n} V_{n}+\sum_{i=1}^{n-2} \lambda_{n} \varepsilon_{n-1} \varepsilon_{n-(i+2)} H_{i}^{*} V_{n-(i+1)}
$$

or

$$
X=\lambda_{n} V_{n}+\lambda_{n} \varepsilon_{n-1} \sum_{i=3}^{n} \varepsilon_{n-i} H_{i-2}^{*} V_{n-(i-1)}
$$

where $\lambda_{n}$ is constant. We will show that it is parallel along $\alpha$; that is, $\nabla_{V_{1}} X=0$. By direct calculation, we have

$$
\begin{aligned}
\nabla_{V_{1}} X= & \nabla_{V_{1}}\left(\lambda_{n} V_{n}\right)+\lambda_{n} \varepsilon_{n-1} \sum_{i=3}^{n} \varepsilon_{n-i} \nabla_{V_{1}}\left(H_{i-2}^{*} V_{n-(i-1)}\right) \\
= & \lambda_{n} \nabla_{V_{1}} V_{n} \\
& +\lambda_{n} \varepsilon_{n-1} \sum_{i=3}^{n} \varepsilon_{n-i}\left[H_{i-2}^{* \prime} V_{n-(i-1)}+H_{i-2}^{*} \nabla_{V_{1}}\left(V_{n-(i-1)}\right)\right] \\
= & \lambda_{n} \varepsilon_{n-1}\left[\begin{array}{c}
-\varepsilon_{n-2} k_{n-1} V_{n-1} \\
+\left(\sum_{i=3}^{n-1} \varepsilon_{n-i} H_{i-2}^{* \prime} V_{n-(i-1)}\right. \\
-\varepsilon_{n-(i+1)} k_{n-i} V_{n-i} H_{i-2}^{*} \\
\left.+k_{n-(i-1)} V_{n-(i-2)} H_{i-2}^{*} \varepsilon_{n-i}\right) \\
\left.+\varepsilon_{0} H_{n-2}^{* \prime} V_{1}+\varepsilon_{0} k_{1} H_{n-2}^{*} V_{2}\right]
\end{array}\right.
\end{aligned}
$$

Here, in the case $n=3$, we omit the term of sum.

On the other hand, by using (9), we can write

$$
\varepsilon_{n-(i+1)} \varepsilon_{n-i} H_{i-2}^{* \prime}=\varepsilon_{n-(i+1)} \varepsilon_{n-i} k_{n-(i-1)} H_{i-3}^{*}-k_{n-i} H_{i-1}^{*}
$$

for $4 \leq i \leq n-1$ together with (11). Moreover, from Lemma 6 , we know that

$$
H_{n-2}^{* \prime}=k_{1} H_{n-3}^{*} \text {. }
$$

Therefore, by using (11), (53), and (54) and by the definition of $H_{1}^{*}$, algebraic calculus shows that $\nabla_{V_{1}} X=0$. Besides, $g\left(V_{n}, X\right)=\lambda_{n} \varepsilon_{n-1}$ is constant. Consequently, $\alpha$ is a $V_{n}$-slant helix in $M$.

Corollary 9. Let $(M, g)$ be a n-dimensional pseudoRiemannian manifold and let $\alpha(s)$ be a proper curve of order $n$ (non-null). Then, $\alpha$ is a $V_{n}$-slant helix in $M$ if and only if $H_{n-2}^{* \prime}=k_{1} H_{n-3}^{*}$ and $H_{n-2}^{*} \neq 0$, where $\left\{H_{1}^{*}, H_{2}^{*}, \ldots, H_{n-2}^{*}\right\}$ denote the harmonic curvature functions of the curve $\alpha$.

Proof. It is obvious by using Lemma 6. and Theorem 8 .

\section{Conclusion}

In this work, $V_{n}$-slant helix is defined and new characterizations are given about the helix by using the harmonic curvature functions in pseudo-Riemannian manifolds. Links with other disciplines of the curve can be examined since the curve is important for other branches of science such as molecular biology and mathematical physics. On the other hand, the curve can be analyzed in different spaces of mathematics.

\section{Conflict of Interests}

The authors declare that there is no conflict of interests regarding the publication of this paper.

\section{Acknowledgment}

The authors would like to express their sincere gratitude to the referees for valuable suggestions to improve the paper.

\section{References}

[1] A. Jain, G. Wang, and K. M. Vasquez, "DNA triple helices: biological consequences and therapeutic potential," Biochimie, vol. 90, no. 8, pp. 1117-1130, 2008.

[2] Y. Yin, T. Zhang, F. Yang, and X. Qiu, "Geometric conditions for fractal super carbon nanotubes with strict self-similarities," Chaos, Solitons and Fractals, vol. 37, no. 5, pp. 1257-1266, 2008.

[3] G. Scarr, "Helical tensegrity as a structural mechanism in human anatomy," International Journal of Osteopathic Medicine, vol. 14, no. 1, pp. 24-32, 2011.

[4] M. A. Lancret, "Memoire sur les courbes a double courbure," Mémoires Présentés à l'Institut, vol. 1, pp. 416-454, 1806.

[5] D. J. Struik, Lectures on Classical Differential Geometry, Dover, New York, NY, USA, 1988.

[6] E. Özdamar and H. H. Hacisalihoğlu, "A characterization of inclined curves in Euclidean $n$-space," Communications de la Faculté des Sciences de l'Université d'Ankara. Series A1, vol. 24, no. 3, pp. 15-22, 1975.

[7] S. Izumiya and N. Takeuchi, "New special curves and developable surfaces," Turkish Journal of Mathematics, vol. 28, no. 2, pp. 531-537, 2004.

[8] M. Önder, M. Kazaz, H. Kocayiğit, and O. Kiliç, “ $B_{2}$-slant helix in Euclidean 4-space $E^{4}$," International Journal of Contemporary Mathematical Sciences, vol. 3, no. 29-32, pp. 1433-1440, 2008.

[9] İ. Gök, Ç. Camci, and H. H. Hacisalihoğlu, " $V_{n}$-slant helices in Euclidean $n$-space $E^{n}$," Mathematical Communications, vol. 14, no. 2, pp. 317-329, 2009.

[10] İ. Gök, C.. Camc1, and H. H. Hacısalihoğlu, " $V_{n}$-slant helices in Minkowski $n$-space $E_{1}^{n}$," Communications de la Faculté des Sciences de l'Université d'Ankara. Séries A1, vol. 58, no. 1, pp. 2938, 2009. 
[11] A. T. Ali and R. López, "Some characterizations of inclined curves in Euclidean $E^{n}$ space," Novi Sad Journal of Mathematics, vol. 40, no. 1, pp. 9-17, 2010.

[12] A. T. Ali and M. Turgut, "Some characterizations of slant helices in the Euclidean space $E^{n}$," Hacettepe Journal of Mathematics and Statistics, vol. 39, no. 3, pp. 327-336, 2010.

[13] M. Külahc1, M. Bektaş, and M. Ergüt, "On harmonic curvatures of a Frenet curve in Lorentzian space," Chaos, Solitons and Fractals, vol. 41, no. 4, pp. 1668-1675, 2009.

[14] S. Özkaldı, İ. Gök, Y. Yaylı, and H. H. Hacisalihoğlu, " $L C$ slant helix on hypersurfaces in Minkowski space $E_{1}^{n+1}$," TWMS Journal of Pure and Applied Mathematics, vol. 1, no. 2, pp. 137145, 2010.

[15] Ç. Camcı, K. İlarslan, L. Kula, and H. H. Hacısalihoğlu, "Harmonic curvatures and generalized helices in $E^{n}$," Chaos, Solitons and Fractals, vol. 40, no. 5, pp. 2590-2596, 2009.

[16] A. Şenol, E. Ziplar, Y. Yayli, and İ. Gök, "A new approach on helices in Euclidean $n$-space," Mathematical Communications, vol. 18, no. 1, pp. 241-256, 2013.

[17] B. O'Neill, Semi-Riemannian Geometry with Applications to Relativity, Academic Press, New York, NY, USA, 1983.

[18] H. H. Song, "On proper helices in pseudo-Riemannian submanifolds," Journal of Geometry, vol. 91, no. 1-2, pp. 150-168, 2008. 


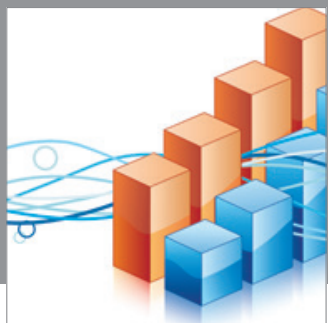

Advances in

Operations Research

mansans

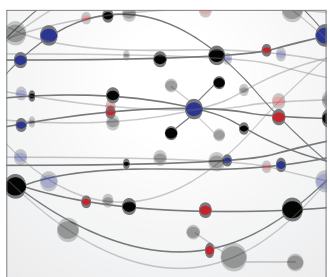

The Scientific World Journal
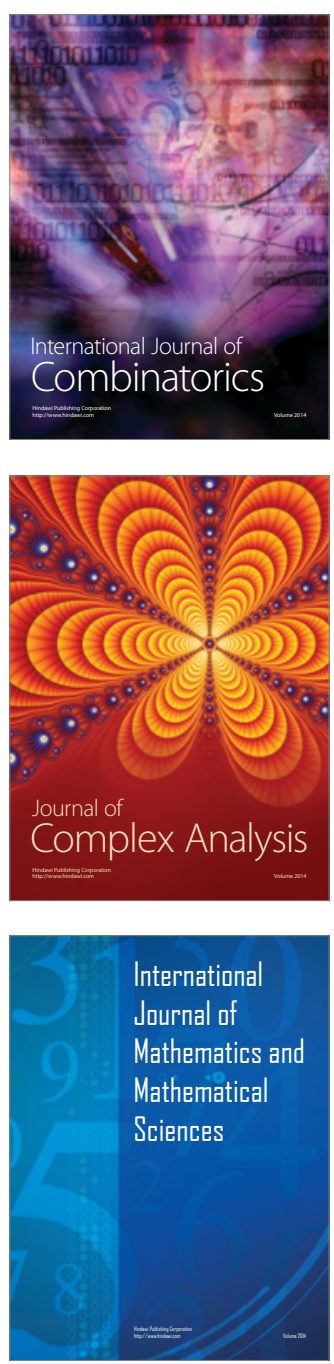
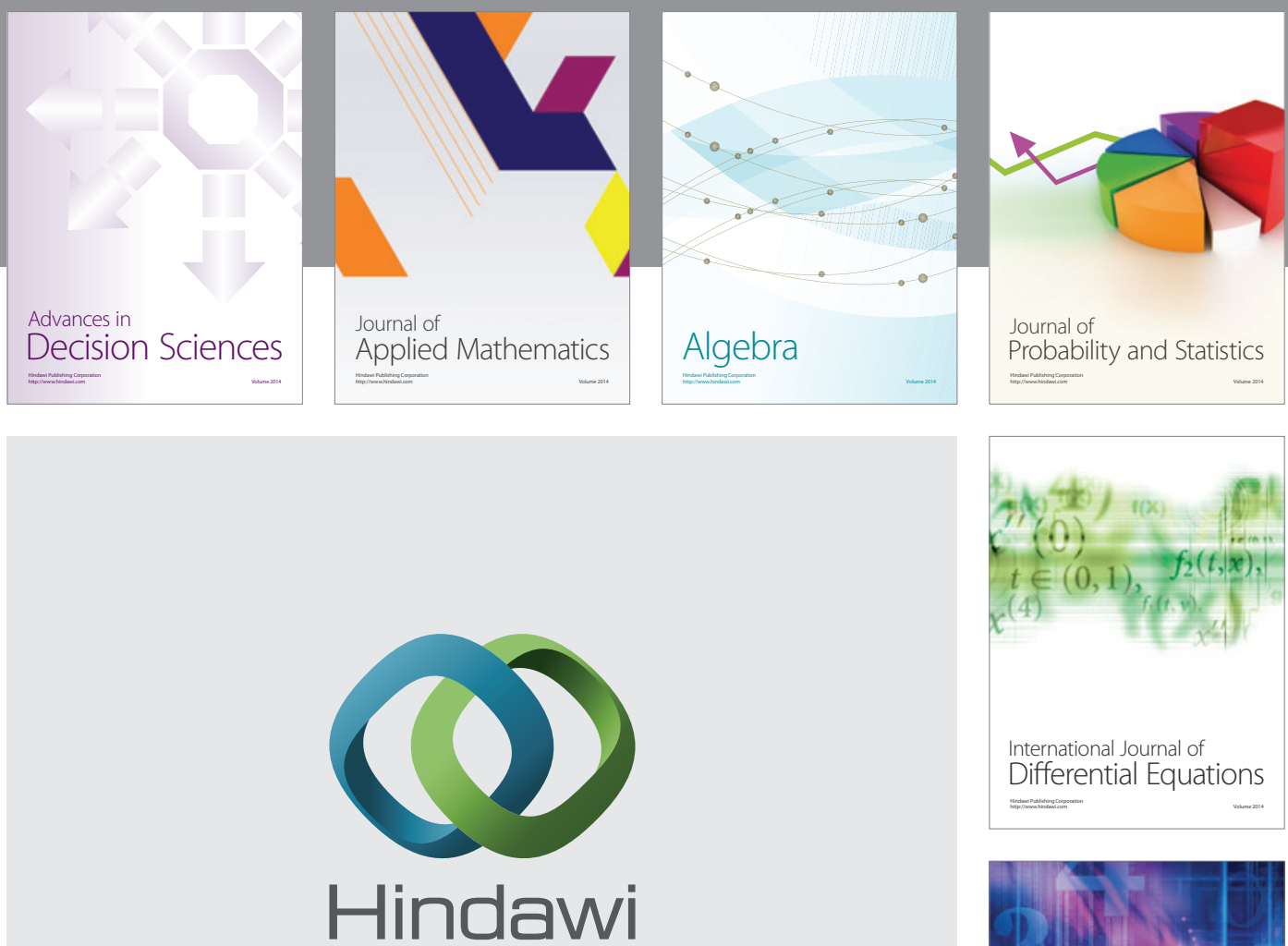

Submit your manuscripts at http://www.hindawi.com
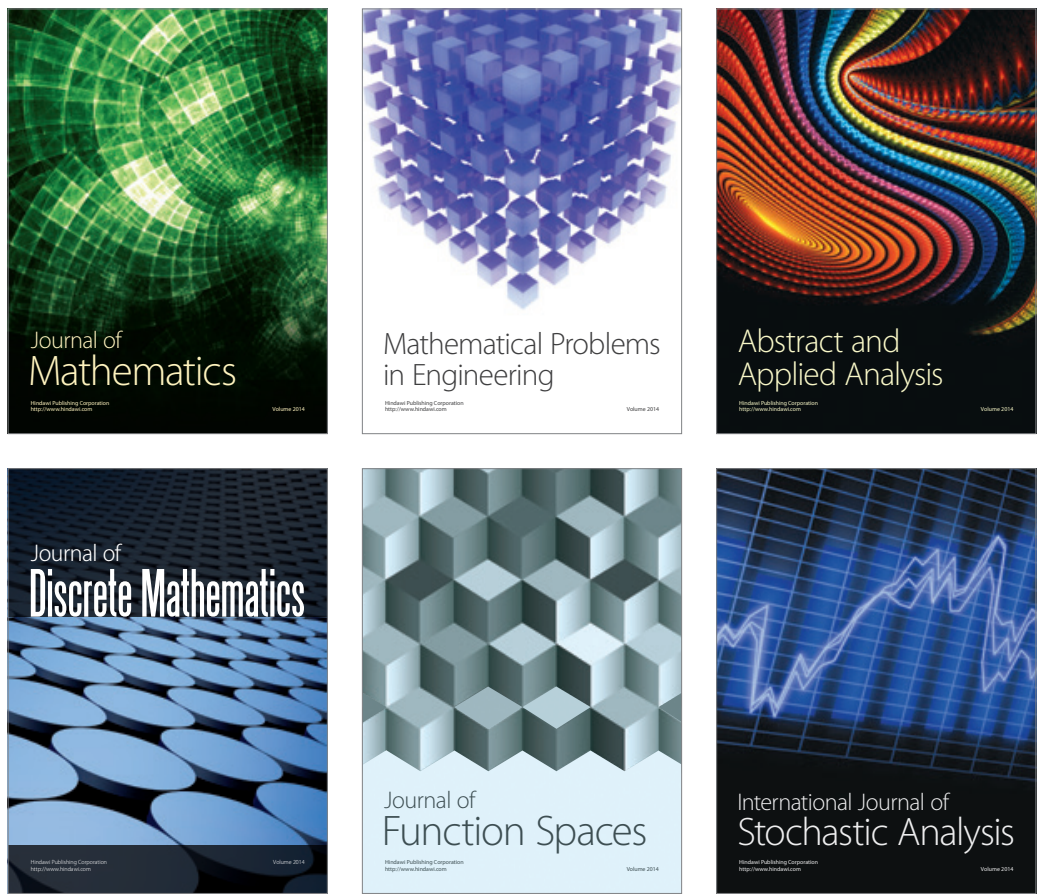

Journal of

Function Spaces

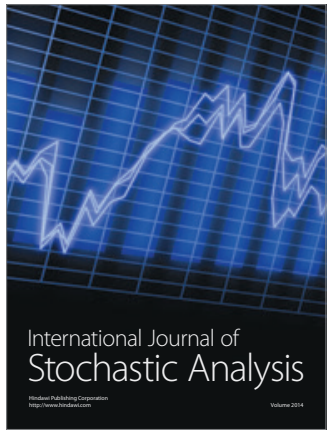

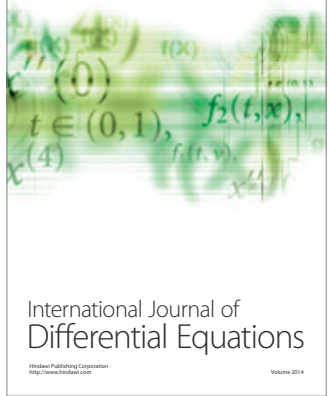
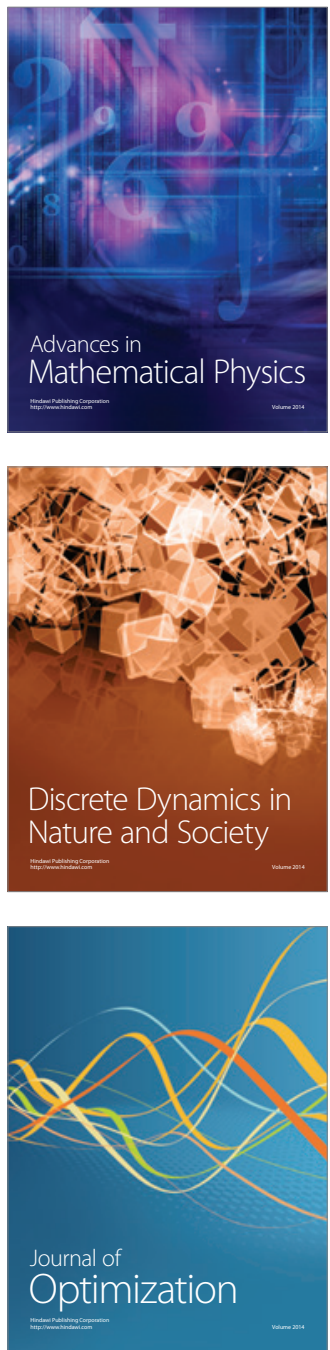